\title{
Evaluación del currículum real de la carrera Administración Turística y Hotelera
}

\author{
MSc. María Catalina Tapia López \\ Docente Investigadora \\ UNAN-MANAGUA, FAREM-CARAZO \\ kathytapia7@hotmail.com
}

MSc. Raúl Alberto Medrano Chávez

Docente Investigador

UNAN-MANAGUA, FAREM-CARAZO

raulunancarazo@hotmail.com

\section{RESUMEN}

a UNAN-Managua ha venido realizando un proceso de análisis y reflexión permanente sobre su quehacer, con el propósito de plantear, desarrollar, evaluar y gestionar. Con el fin de alcanzar niveles de excelencia en los procesos académicos, se vuelve imprescindible, entre otras actividades, la evaluación permanente del currículum, a fin de poder mejorarlo de manera continua, tal como señala Brovelli (2001:102), "Entender que evaluar el currículum y las instituciones educativas es comenzar a mejorarlas...." Asimismo, el proceso de evaluación y actualización del currículum permite incorporar los avances que se dan en el campo de la ciencia y la tecnología y de esta forma, poder contribuir a la solución eficiente y oportuna a los problemas de entorno.

\section{INTRODUCCIÓN}

La globalización y los avances vertiginosos en el campo de la tecnología y las comunicaciones han propiciado el desarrollo de la sociedad del conocimiento, que trae consigo nuevos retos y desafíos que deben ser asumidos por todas las instituciones educativas, de manera particular, por las de educación superior, a fin de garantizar la formación de profesionales que posean las competencias necesaria para dar respuestas oportunas a las demandas y necesidades de su entorno.

En ese sentido, es de vital importancia que las universidades evalúen y ajusten de manera permanente su currículo para que este incorpore, fundamentalmente, los avances que se susciten en el campo de la tecnología y las ciencias, mediante estrategias didácticas innovadoras, que permitan a las instituciones educativas satisfacer las demandas de la sociedad y cumplir su misión con calidad.

La Universidad Nacional Autónoma de Nicaragua (UNAN-Managua) se encuentra inmersa en un proceso de acreditación universitaria, proponiéndose un conjunto de acciones estratégicas encaminadas a lograr esta meta, que permitan elevar los estándares de calidad, tanto a nivel 
académico como de gestión. Es por ello que se considera pertinente, promover procesos permanentes de evaluación del currículo, a fin de alcanzar estos estándares de calidad.

El presente trabajo se establece como un primer esfuerzo para promover estos procesos permanentes de evaluación curricular en la UNAN-Managua, iniciando en un primer momento en la Facultad Regional Multidisciplinaria de Carazo (FAREM-Carazo). Para este propósito se ha seleccionado la Carrera Licenciatura en Administración Turística y Hotelera, que fue gestada en esta facultad, adscrita al Departamento de Ciencias Económicas y Administrativas. La Carrera cuenta con cuatro grupos de clases, con una población de 110 estudiantes, de primero, tercero, cuarto y quinto año; con una perspectiva de egresados del 20 \% para el año 2015.

El currículo de la carrera fue preparado por una Comisión Curricular de Carrera conformada por docentes de la FAREM-Carazo, estructurada de la siguiente manera: un coordinador, cinco docentes con formación y experiencia, tanto en el área administrativa como turística, un representante del gremio de los trabajadores docentes, un representante de la dirigencia estudiantil y la dirección del departamento de Ciencias Económicas y Administrativas.

El currículo fue diseñado tomando como base el Modelo Educativo, Normativa y Metodología para la Planificación Curricular 2011 de la UNAN-Managua, que es un modelo centrado en el estudiante y en el desarrollo de procedimientos, habilidades, estrategias y técnicas, que les permitan aprender a ser, conocer, hacer, convivir, emprender y crear iniciativas que impulsen el sector turístico y hotelero en la región y el país, utilizando el eje de investigación como un componente esencial del proceso de educación, mediante la metodología del aprendizaje por descubrimiento dentro y fuera del aula de clases. Todo el proceso de elaboración contó con el acompañamiento y asesoría de especialistas de la Dirección Académica de la UNAN-Managua.

Para alcanzar niveles de excelencia en los procesos académicos, se vuelve imprescindible, entre otras actividades, la evaluación permanente del currículum, a fin de poder mejorarlo de manera continua, tal como señala Brovelli (2001:102), "Entender que evaluar el currículum y las instituciones educativas es comenzar a mejorarlas...”.

Casarini (2009) señala, que existen diferentes parcelas del currículum que son susceptibles a la evaluación: el currículum formal, donde se evalúa el perfil del egresado y la estructura del plan de estudio; el currículum real, que se centra en la evaluación de las estrategias aplicadas por los docentes, los procesos de aprendizaje, los productos del aprendizaje, así como las condiciones materiales, espaciales, humanas y, finalmente, el currículum oculto que conlleva la evaluación de los valores, modelos, actitudes de los maestros y alumnos, los procesos de socialización en el aula, entre otros aspectos.

\section{METODOLOGÍA}

Se tomó como punto de partida lo expuesto por Casarini (2009) que señala, que el currículum real es la puesta en práctica del currículo formal con las inevitables y necesarias modificaciones que requiere la contrastación y ajuste entre un plan curricular y la realidad del aula, sin perder de vista que el currículum real encuentra su razón de ser en la práctica educativa.

Se ha considerado, que los docentes son una fuente de información primordial para la evaluación de currículum real. Casarini (2009) señala, que "Los maestros constituyen un sector privilegiado 
en la evaluación curricular y la enseñanza, por lo cual, es saludable considerar, por una parte, que ellos son informantes clave en los procesos evaluativos..." ( $p$ 195). En este proceso, se aplicaron encuestas a cinco docentes que imparten clases en la Carrera en estudio.

Asimismo, los estudiantes constituyen otra fuente de información primordial para el diagnóstico. Casarini afirma, que las figuras cruciales en el funcionamiento del currículum son los docentes y estudiantes y constituyen la preocupación más importante del evaluador del currículum. Para este estudio se aplicó encuesta a 52 estudiantes de tercero, cuarto y quinto año de la Carrera.

Adicionalmente, los coordinadores de Carrera han sido considerados como fuente de información importante para este estudio, ya que ellos tienen definido, entre sus funciones, coordinar el trabajo académico que realizan los docentes. En este sentido, se entrevistó a los dos coordinadores que tienen asignada la carrera de Administración Turística y Hotelera.

Por otra parte, Brovelli (pág. 114) expone, que la evaluación del currículum debe tomar en cuenta la valoración externa, para esta se toma en cuenta a un grupo focal de cuatro egresados, con la finalidad de indagar acerca del tipo de funciones profesionales que realmente desempeñan, conocer acerca de la calidad de su desempeño, de las competencias adquiridas, durante su formación y las puestas en práctica.

El procesamiento de la información se realizó utilizando el paquete estadístico SPSS v.18, particularmente para el procesamiento de las encuestas aplicadas a los estudiantes. En el caso de la información recopilada a partir de las entrevistas y el grupo focal, se diseñaron tablas a fin de facilitar su proceso análisis.

\section{RESULTADOS Y DISCUSIÓN}

A continuación, se presentan los principales hallazgos del proceso de evaluación del currículo real de la Carrera Licenciatura en Administración Turística y Hotelera (ATH), en la FAREMCarazo. Inicialmente, se muestran los resultados derivados de preguntas generales realizadas a los estudiantes. Luego, retomando lo señalado por Casarini, se organizan los resultados considerando los aspectos cuando se evalúa el currículo real: evaluación de estrategias aplicadas por los docentes, evaluación de los procesos de aprendizaje, la evaluación de los productos de aprendizaje, así como la evaluación de las condiciones materiales, espaciales, humanas, entre otras. En estos acápites, se presenta el cruce de opiniones, tanto de los estudiantes como de los docentes, así como la opinión brindada por los coordinadores de Carrera.

\subsection{Información de la carrera}

De manera inicial se preguntó a los estudiantes si conocían el plan de la Carrera; tan solo el 35 \% de los informantes expresaron conocerlo. Un dato parcialmente positivo es que la Carrera poco a poco va logrando atractivo en el mercado académico (estudiantes de secundaria) considerando el auge y crecimiento que experimenta el sector turístico en nuestro país. Esto se evidencia en la encuesta aplicada, en la que el $75 \%$ de los estudiantes afirmaron que esta Carrera fue su primera opción de ingreso en la Universidad. Este dato se contrapone con las estadísticas del año 2010, en la cual solo un 10 \% de los estudiantes de primer año de la Carrera habían expresado que era su primera opción.

Al preguntar a los docentes que imparten clases en la Carrera de Administración Turística y Hotelera 
acerca del perfil de esta, todos coinciden en conocerlo, además externaron que, de manera general, los contenidos plasmados permiten alcanzar los objetivos generales del programa; sin embargo, expusieron que existen programas que omiten temas que son de relevancia para la asignatura, por lo que en ocasiones realizan adecuaciones a fin de fortalecer los conocimientos de los estudiantes y que muchas veces, el número de horas asignado es insuficiente para el desarrollo de todos los contenidos.

\subsection{Evaluación de las estrategias aplicadas por el docente}

Para la evaluación de las estrategias de aprendizaje, se tomó en consideración una serie de factores organizados en dos categorías: intervención del docente y estrategias didácticas utilizadas por el docente.

\subsubsection{Intervención del docente}

En cuanto a la intervención del docente en el aula de clase se conoció, que un porcentaje menor al $50 \%$ de los docentes, explican siempre a sus estudiantes los objetivos de la asignatura y que un $25 \%$ a veces lo hacen. (Ver gráfico 1)

\section{Al inicio de la clase el docente explica los objetivos de la asignatura}

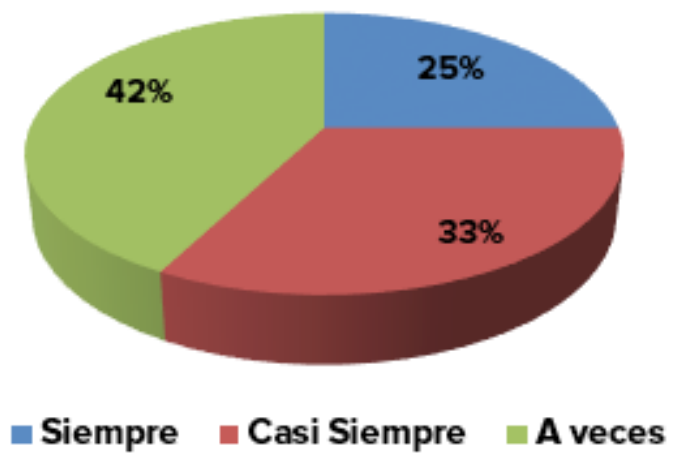

Grafico 1. Explicación de los objetivos de la asignatura

Lo anterior evidencia, que en el desarrollo de algunas asignaturas, los estudiantes desconocen los propósitos que se quieren alcanzar y los aportes que la asignatura brinda para el cumplimiento del perfil de la Carrera.

Luego se consultó a los estudiantes si sus docentes explicaban los objetivos de aprendizaje al inicio de cada unidad. (Ver gráfico 2 en página siguiente). 


\section{Al inicio de la unidad el docente orienta los objetivos de aprendizaje}

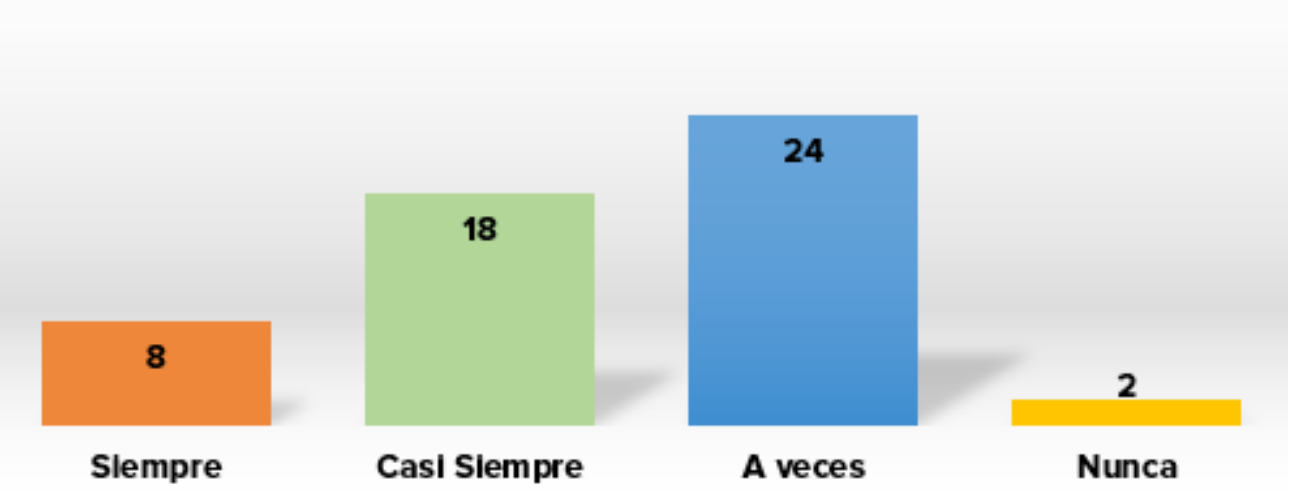

Gráfico 2. Explicación de objetivos por unidad.

El $46 \%$ de los informantes consideran que la mayoría de los docentes no orientan claramente los objetivos de los aprendizajes, lo que no permite una adecuada interiorización de los conocimientos por parte de los estudiantes, ya que al no comprender la utilidad real de los conocimientos y su aplicabilidad, el aprendizaje se vuelve menos significativo.

Por otra parte, solo el $40 \%$ de los consultados opinaron que los docentes siempre preguntan sobre los conocimientos previos de los contenidos que se desarrollarán, lo que evidencia que no todo todos los docentes están realizando las evaluaciones diagnósticas orientadas en el Modelo Educativo y los programas de asignatura, por tanto, se pierde la oportunidad de validar y reconstruir, en caso de ser necesario, los conocimientos previos de los estudiantes.

Un aspecto fundamental en la evaluación real del currículo lo constituye la metodología aplicada por el docente. Se consultó a los estudiantes sobre este aspecto y el 94 \% de los encuestados opinan, que el docente utiliza una metodología adecuada que se ajusta a las características de la asignatura que desarrolla (Ver gráfico 3). Asimismo, el 80 \% considera que los recursos didácticos utilizados por los docentes, son pertinentes para el desarrollo de los contenidos, lo que representa una fortaleza en relación a la intervención del docente en el aula.

\section{La metodología aplicada por el docente va acorde con las características de la asignatura}

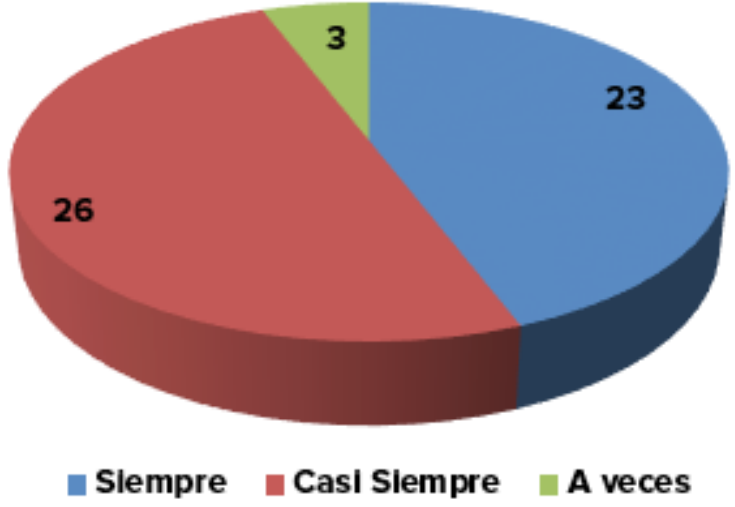

Gráfico 3. Metodología acorde a las características de la asignatura. 
Es importante destacar, que la opinión de los docentes coincide con la percepción de los estudiantes, ya que el $98 \%$ de los docentes consultados opinan, que las actividades metodológicas seleccionadas para el desarrollo de los contenidos son las más adecuadas para alcanzar los aprendizajes propuestos en los objetivos. Además expresaron, que toman como referente la metodología sugerida en el programa de la asignatura, esto evidencia, que los docentes consultados ajustan su práctica docente a las orientaciones metodológicas brindadas en los programas de asignatura.

En relación a las actividades de cierre al final de cada sesión de clases, solo el $23 \%$ de los informantes opinan que los docentes hacen un resumen de los contenidos abordados al finalizar la clase, es decir, no se está realizando el proceso de consolidación de lo aprendido. Por otra parte, el $19 \%$ de los estudiantes expresan que los docentes siempre asignan actividades de estudio independiente. Esta práctica evidencia el no cumplimiento con lo establecido en el modelo curricular y en el plan temático de los programas de asignaturas, ya que en estos se incluye un número significativo de horas para el trabajo independiente de los estudiantes.

El Modelo Educativo de la UNAN-Managua establece, que las Tecnologías de la Información y la Comunicación (TIC) se utilizará, no solo para mejorar las prácticas pedagógicas, sino también para permitir a los estudiantes otros escenarios de aprendizaje. En este sentido, se consultó a los estudiantes acerca del uso de la tecnología por parte de los docentes, solo el $10 \%$ de los encuestados externaron que los docentes hacen uso de la tecnología, esto evidencia el no cumplimiento a lo establecido en el Modelo Educativo. Sin embargo, lo anterior se contradice con lo expuesto por los docentes consultados, ya que ellos afirman que utilizan recursos tecnológicos para el desarrollo de sus asignaturas.

Se consultó a los estudiantes si los docentes orientaban el uso de recursos bibliográficos para el desarrollo de la asignatura. El 93 \% de los encuestados expresaron que los docentes siempre orientan el uso de bibliografía. Al respecto, los docentes dijeron que pocos utilizan la bibliografía sugerida en los programas de asignatura, ya que la consideran insuficiente, planteando la necesidad de actualizarla anualmente.

\subsubsection{Estrategias didácticas utilizadas}

Se consultó a los estudiantes sobre las estrategias didácticas implementadas por sus docentes, obteniendo los resultados que se muestran en el Gráfico 4. (ver gráfico en página siguiente) 


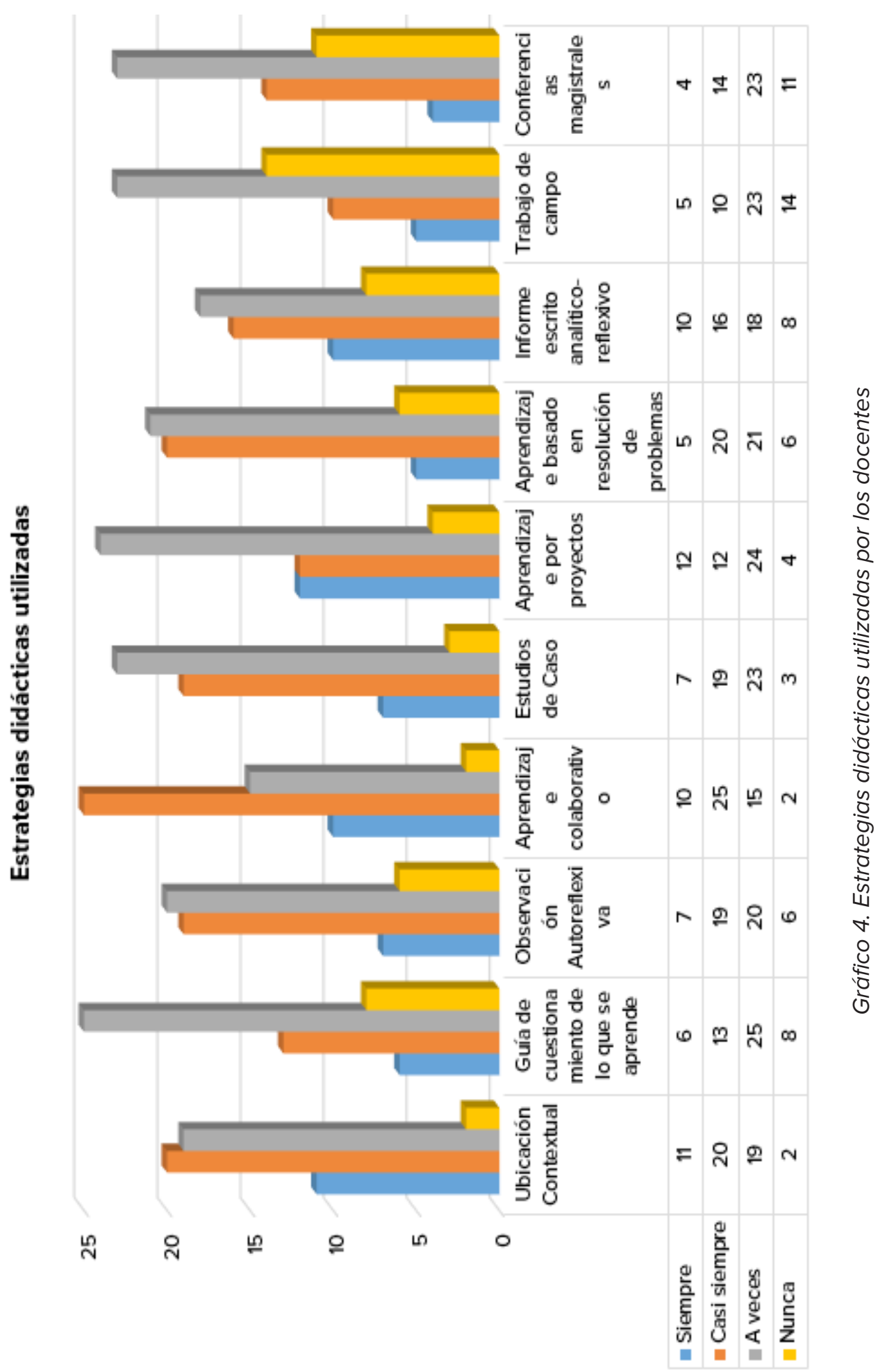

Esto evidencia, que los docentes están implementando en el aula de clase las estrategias didácticas orientadas en el Modelo Educativo de la UNAN-Managua, siendo la ubicación contextual y el aprendizaje colaborativo las estrategias más utilizadas. Resulta muy interesante la coincidencia entre la opinión de los estudiantes y los docentes, ya que ambas fuentes de 
información concuerdan en cuáles son las estrategias más utilizadas. Asimismo, los docentes externaron que el trabajo de campo y el informe escrito analítico-reflexivo, son las estrategias que menos utilizan.

Sin embargo, es vital destacar que los coordinadores de Carrera, salvo en el caso de docentes de reciente contratación, no realizan un acompañamiento metodológico que permita verificar la implementación real de estas estrategias en el aula de clase. Es decir, no se da un seguimiento a la ejecución real de los planes de asignatura semestrales elaborados y entregados por los docentes. Los coordinadores expresaron, que el seguimiento que se da a todos los docentes, es mediante las supervisiones indirectas diarias, en la que el docente informa acerca de la unidad y contenidos a desarrollar. Sin embargo, no se valora cómo se desarrolla ese contenido en el aula de clase sistemáticamente, solo cuando se hacen supervisiones científica-técnica.

\subsection{Evaluación de los procesos de aprendizaje}

Para la evaluación de los procesos de aprendizaje, se tomó en cuenta un conjunto de aspectos que se organizaron en tres categorías: aprendizaje significativo, metodologías participativas y evaluación de la asignatura.

\subsubsection{Aprendizaje significativo}

Los estudiantes consideran que las metodologías que facilitan aprendizaje más significativo son la resolución de casos y los trabajos de campo. En el caso de la resolución de casos afirmaron que les permitía poner en práctica los conocimientos adquiridos y de esta forma, darle un significado a lo que aprenden. De igual forma, consideran que los trabajos de campo que realizan, principalmente a través de las Prácticas de Formación Profesional, le ha permitido vincularse con el campo laboral y adquirir conocimientos que no se pueden obtener en el aula de clase.

Lo expuesto anteriormente es corroborado por los estudiantes egresados de la Carrera, ya que durante el desarrollo del grupo focal, compartieron que mediante la resolución de estudios de caso, debían no solo poner en práctica los conocimientos ya adquiridos, sino que además, debían investigar a fin de poder dar solución a los problemas presentados. Asimismo señalaron, que las asignaturas vinculadas con la investigación y la formulación de proyectos son las que le permitieron desarrollar un aprendizaje más significativo.

\subsubsection{Metodologías participativas}

En lo referente a las metodologías participativas, el Modelo Educativo de la UNAN-Managua señala que el proceso de enseñanza-aprendizaje implementado por esta casa de estudio, tiene como base un modelo pedagógico que centra su atención en el estudiante que asume un rol activo y participativo, por lo que en el modelo se orienta la realización de trabajos a fin de que los estudiantes tengan un rol activo en su proceso de aprendizaje. Según la opinión de los alumnos (72\%), el profesorado está promoviendo las metodologías participativas, lo cual coincide con que una de las estrategias didácticas más utilizada es el aprendizaje colaborativo.

\subsubsection{Procesos de evaluación sumativa}

Se consultó a los estudiantes acerca de los procesos de evaluación sumativa de las asignaturas. Se les preguntó si los docentes explicaban al inicio del curso el sistema de evaluación a implementar 
y si realizaban cuatros trabajos de grupo y cuatro pruebas cortas individuales, tal y como lo establece el Modelo Educativo de la UNAN-Managua. El 89 \% de los consultados respondieron que siempre, o casi siempre, los docentes explican el sistema de evaluación del curso. De igual forma expresaron que el 92 \% de los docentes cumple con la forma de definida en el Modelo Educativo.

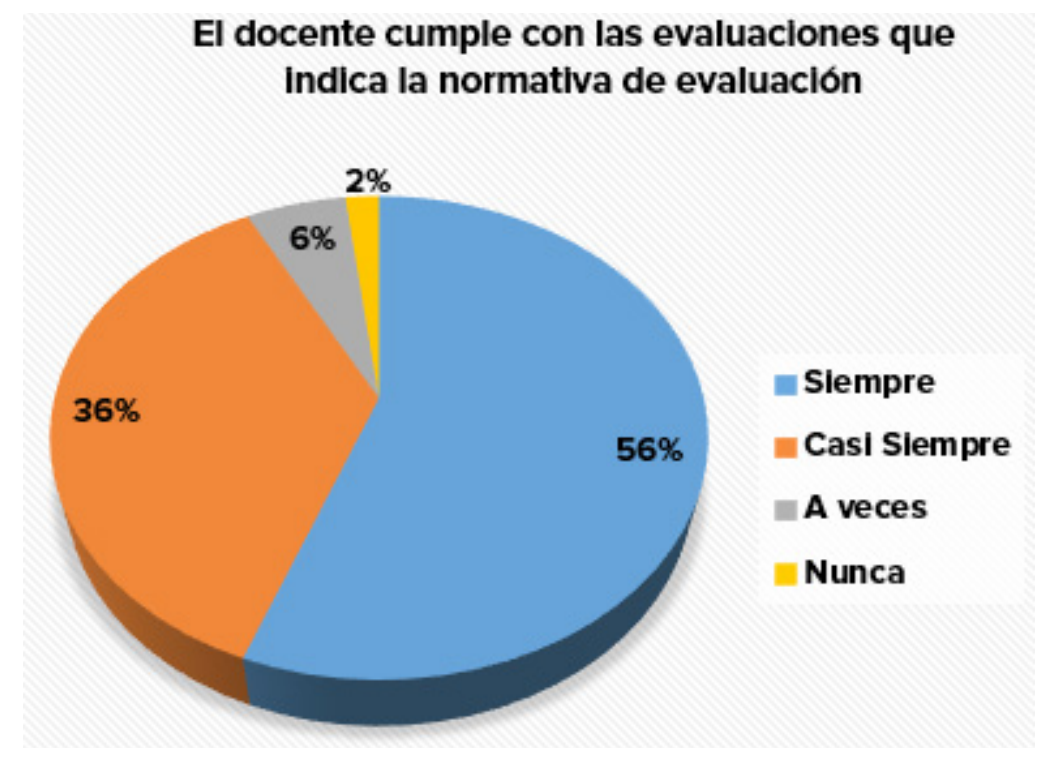

Gráfico 5. Cumplimiento de evaluación sumativa según normativa.

De igual manera, los docentes afirmaron que explican a los alumnos los métodos de evaluación que se utilizarían durante el desarrollo de la asignatura, considerando como referente lo establecido por la normativa de evaluación, cumpliendo incluso con las fechas que proponen la autoridades académicas de la facultad (iniciando en la semana número tres, exámenes en las semanas once y doce, hasta culminar en la semana quince).

Continuando con lo referido al proceso de evaluación, se les consultó a los estudiantes si los docentes entregaban en tiempo y forma los resultados de sus evaluaciones, ya que el reglamento académico establece que deben ser entregados 72 horas después de realizada la actividad evaluativa.

En el gráfico 6 se evidencia que son muy pocos los docentes que entregan en tiempo y forma los resultados de las evaluaciones. Es importante señalar, que muchos estudiantes externaron que varios docentes nunca les entregan los trabajos que realizan y no les informan sobre los acumulados que van obteniendo a lo largo del semestre. 


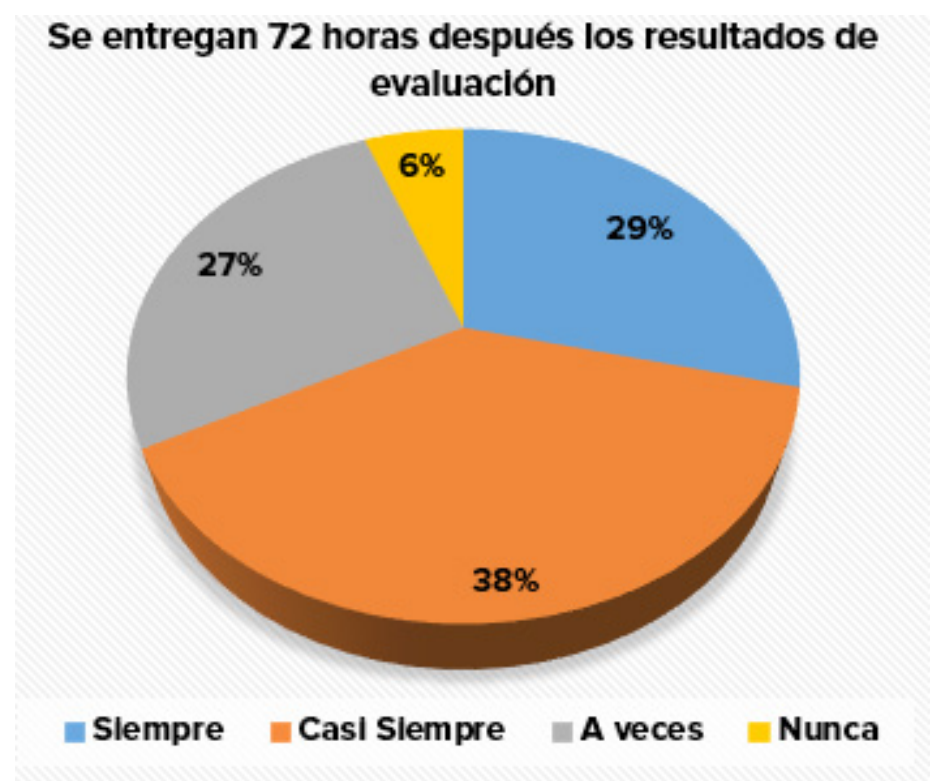

Gráfico 6. Entrega de resultados de evaluación según reglamento

Además, la mayoría de los estudiantes consultados afirman que no reciben ningún tipo de retroalimentación de sus trabajos, pruebas cortas y exámenes (ver gráfico 7), esto representa una afectación directa a la calidad educativa y por tanto, el incumplimiento de lo establecido en el Modelo Educativo y currículo de la carrera, ya que se le limita al estudiante la oportunidad de aprender a partir de sus errores, quedando con vacíos a lo largo de su proceso de formación.

\section{El docente realiza retroalimentación}

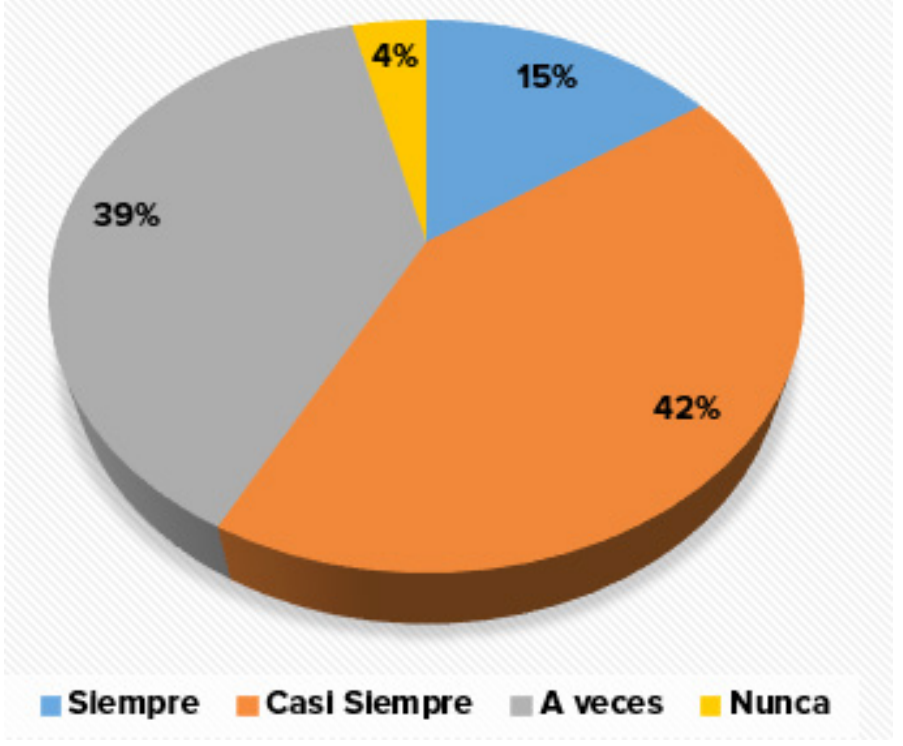

Gráfico 7. El docente realiza retroalimentación

Por su parte, los docentes, a diferencia de los alumnos, expresaron que cumplen con la entrega de las calificaciones de las distintas evaluaciones en tiempo y forma, pero coincidieron con los estudiantes en que a veces no se realiza la retroalimentación de las evaluaciones, argumentando limitantes de tiempo. 
Con respecto a las evaluaciones, el $55 \%$ de los estudiantes mencionaron que los métodos y técnicas utilizadas para evaluar las unidades fueron los más adecuados, sin embargo, el 45\% considera que no es así, principalmente en lo referido a las pruebas cortas. En este punto es importante señalar, que al momento de entrevistar a los coordinadores de la Carrera, quedó en evidencia que los docentes no presentan las pruebas cortas que aplican a sus estudiantes ni las rúbricas donde se definan los criterios a evaluar en los trabajos asignados o en los proyectos de fin de curso.

\subsection{Evaluación de los productos de aprendizaje}

Los coordinadores externaron que en el 2014, dos equipo de estudiantes del $\mathrm{V}$ año participaron en la Jornada de Innovación y Emprendimiento a nivel de la Facultad, clasificando uno de estos grupos para participar a nivel nacional en el concurso de innovación, promovido por CONICYT y el CNU. Estos estudiantes obtuvieron el segundo lugar a nivel nacional en la rama de protección al Medio Ambiente.

Estudiantes de quinto año presentaron 15 informes de Formulación y Evaluación de Proyectos vinculados al sector turístico y hotelero. Además, estudiantes de los años superiores presentaron en el 2014, diez proyectos que participaron en la Jornada Universitaria de Desarrollo Científico (JUDC).

Por otra parte, en el año 2014, estudiantes de quinto año de la Carrera realizaron prácticas profesionales en diferentes entidades turísticas. En el año 2015, se ubicaron todos los estudiantes de tercero, cuarto y quinto año de la Carrera, en diferentes organizaciones turísticas y hoteleras. Esto les ha permitido la puesta en práctica de los conocimientos adquiridos a lo largo de su Carrera, obteniendo los estudiantes experiencias muy positivas. Asimismo, gracias al vínculo que la Facultad tiene con INTUR, 20 estudiantes de la Carrera participaron de manera activa en el plan verano 2014 y 2015, apoyando como guías turísticos en el departamento de Carazo.

\subsection{Evaluación de las condiciones materiales}

\subsubsection{Condiciones del aula}

Con respecto a las condiciones del aula, el $90 \%$ de los estudiantes encuestados valoraron entre excelente y muy buena la correspondencia entre el tamaño del aula y el número de estudiantes, la iluminación y ventilación de las mismas, así como el aislamiento de factores externos como el ruido. En estos aspectos, tanto los docentes como los coordinadores de Carrera, coincidieron con la percepción de los estudiantes, quienes valoran positivamente las instalaciones en las cuales se desarrollan las clases.

\subsubsection{Laboratorios de Computación}

La Facultad cuenta con cuatro laboratorios de informática modernos, equipados con 120 equipos que tienen instalados los programas básicos y la conexión a Internet, a fin de garantizar las condiciones óptimas que permitan a los estudiantes el desarrollo de competencias básicas en informática y, a la vez, utilicen la computadora en el desarrollo de las competencias requeridas. Sin embargo, es vital destacar que estos laboratorios no están ubicados en el edificio donde los estudiantes reciben clase, lo que en muchos casos se dificultad el acceso a los mismo, debido a que deben trasladarse de edificio para poder tener acceso a los mismos. 


\subsubsection{Laboratorios de idiomas}

Se cuenta con un moderno laboratorio de idiomas, equipado con dispositivo multimedia especializado para su fin, pero lamentablemente, los estudiantes de ATH lo visitan con muy poca frecuencia, debido a que los docentes no programan uso del mismo. Esta situación representa una gran debilidad, más aun, considerando el perfil de la Carrera, la cual establece que los egresados deben graduarse dominando el idioma inglés.

\subsubsection{Biblioteca}

Se cuenta con una biblioteca, pero en algunas asignaturas se carece de la bibliografía necesaria, lo que obliga a los estudiantes a recurrir a la investigación en Internet. Es importante mencionar, que muy pocos docentes orientan hacer usos de motores de búsqueda en revistas indexadas. Como debilidad se menciona, la poca afluencia de docentes y estudiantes a la biblioteca de la Facultad, esto afecta directamente la calidad del proceso educativo.

\subsubsection{Recursos varios}

La Facultad cuenta con medios de transporte que pueden ser utilizados por los estudiantes para realizar visitas de estudio. Sin embargo, hay momentos que no están disponibles, ya que han sido reservados para otras actividades de la Universidad o por estudiantes de otras Carreras.

En el caso de primer año, se necesita contratar buses externos por la cantidad de estudiantes. Además, los estudiantes deben asumir el costo del combustible, lo que en ocasiones limita la realización de visitas de campo, dado que muchos de los estudiantes de la Facultad son de escasos recursos económicos.

De manera general, los docentes cuentan con pocas oficinas, mobiliario y equipos de cómputo, acceso a Internet. Por otra parte, la Facultad solo cuenta con cinco proyectores multimedia (data show) en excelente condiciones y tres, en proceso de reparación, los cuales resultan insuficientes para la alta demanda de los docentes, esto genera que poco de ellos puedan utilizarlos en el desempeño de sus funciones académicas, restringiendo al docente en el desarrollo de sus estrategias didácticas.

Es importante destacar, que las diferentes fuentes consultadas, entre estos los egresados y los coordinadores de Carrera, expusieron la necesidad de contar con un laboratorio de alimentos y bebidas, que permita la implementación de metodologías participativas en diferentes asignaturas fundamentales de la Carrera, que demandan de este tipo de espacios.

En muchas de estas clases, los estudiantes realizan prácticas básicas relacionadas a la manipulación de alimentos y bebidas, improvisando en el aula, sin embargo, resultan insuficientes.

\section{CONCLUSIONES}

Culminado el proceso de recopilación y análisis de la información, derivamos las siguientes conclusiones:

- Al inicio de cada semestre, los docentes entregan el plan semestral de las asignaturas a impartir. Los coordinadores de Carrera validan la concordia existente entre este plan y el programa de la asignatura, verificando además, que los docentes propongan la implementación de las 
estrategias didácticas definidas en el Modelo Educativo de la UNAN-Managua. Sin embargo, no se da un seguimiento a la ejecución real de ese plan, en el aula de clase.

- Un hallazgo importante es que se da poco acompañamiento y supervisión metodológica a los docentes, ya que esta generalmente se realiza con los docentes contratados como horarios, docentes noveles y docentes que presentan debilidades en su desempeño (quejas de los alumnos, incumplimiento en la entrega de planes, avances e informes).

- Se valora de manera parcial el desarrollo del currículo real con respecto al currículo formal en las diferentes asignaturas, mediante las supervisiones indirectas diarias, en la que el docente informa acerca de la unidad y contenidos a desarrollar. Sin embargo, no se evalúa cómo se desarrolla ese contenido en el aula de clase sistemáticamente, solo cuando se hacen supervisiones científica-técnica.

- De manera general, los contenidos orientados en los programas de asignatura permiten alcanzar los objetivos generales del programa y el perfil de la Carrera. Aunque, existen programas que no incorporan temas que son de vital importancia para la asignatura, por lo que en ocasiones, los docentes realizan adecuaciones a fin de fortalecer los conocimientos de los estudiantes. Además, se presenta debilidades en el perfil turístico por carencias de asignaturas propias de la industria turística.

- Se da poca realización de evaluaciones diagnósticas por parte de los docentes, lo que no permite atribuir un sentido y construir los significados implicados de los contenidos; también, se carece de un adecuado proceso de retroalimentación por parte del docente. Así mismo, se da poco seguimiento y verificación de los métodos de evaluaciones aplicadas por el docente.

- Los docentes hacen poco uso de las Tecnologías de la Información y Comunicación (TIC) en el proceso de enseñanza-aprendizaje, es bajo el aprovechamiento de los laboratorios de inglés y de informática por parte de los estudiantes de la Carrera. La infraestructura con que cuenta la Universidad es insuficiente para el desarrollo de asignaturas propias de la Carrera, ya que se carece de un laboratorio de alimentos y bebidas, así como un laboratorio de hospedería y restaurante.

\section{REFERENCIAS BIBLIOGRÁFICAS}

Brovelli, M. (2001). Evaluación curricular. Fundamentos en Humanidades , 101-122.

Casarini, M. (2009). Teoría y Diseño Curricular. México: Trillas.

Castañeda, M. T., Castro, F. \& Mena, C. (2012). Instrumentos para evaluar el curriculum formal en carreras pedagógicas. Panorama, 6 (10) , 71-85.

Cervantes, E. (25 de Septiembre de 2009). Slideshare. Recuperado el 12 de Junio de 2015, de http://es.slideshare.net/ guestdb53ce/los-cuadernos-de-losalumnos-una-evaluacin-del-currculo-real

Di Franco, G., Siderac, S. \& Di Franco, N. (4 de Noviembre de 2004). El curriculum real y la formación docente. Obtenido de: http:// www.uccor.edu.ar/paginas/encuentro1/ difranco.D.pdf

Perrenoud, P. (1990). Cap.8: El curriculum real y el trabajo escolar en la construcción del éxito y del fracaso escolar. (págs. 213-222). Madrid: Morata. 
UNAN-Managua. (2015). Proyecto Institucional. Managua: Editorial Universitaria.

Universidad Nacional Autónoma de Nicaragua, M. (2011). Modelo Educativo, Normativa y Metodología para la Planificación Curricular 2011. Managua: Editorial Universitaria.

\section{ANEXO. EVIDENCIA FOTOGRÁFICA}
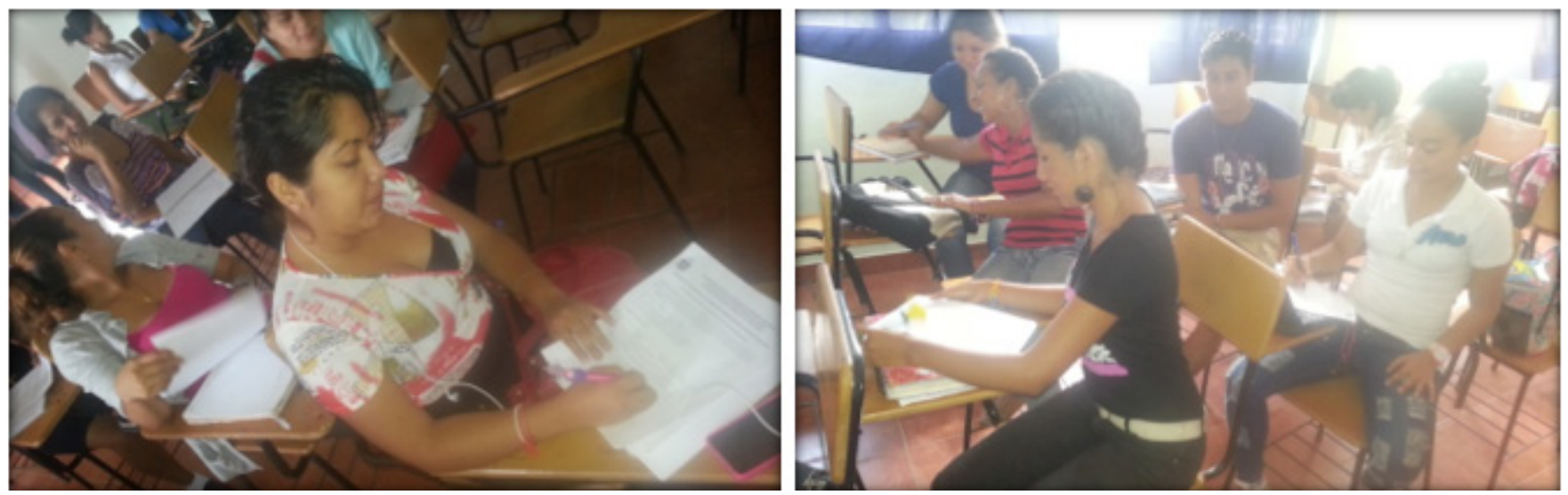

Fotografía de estudiantes llenando encuestas
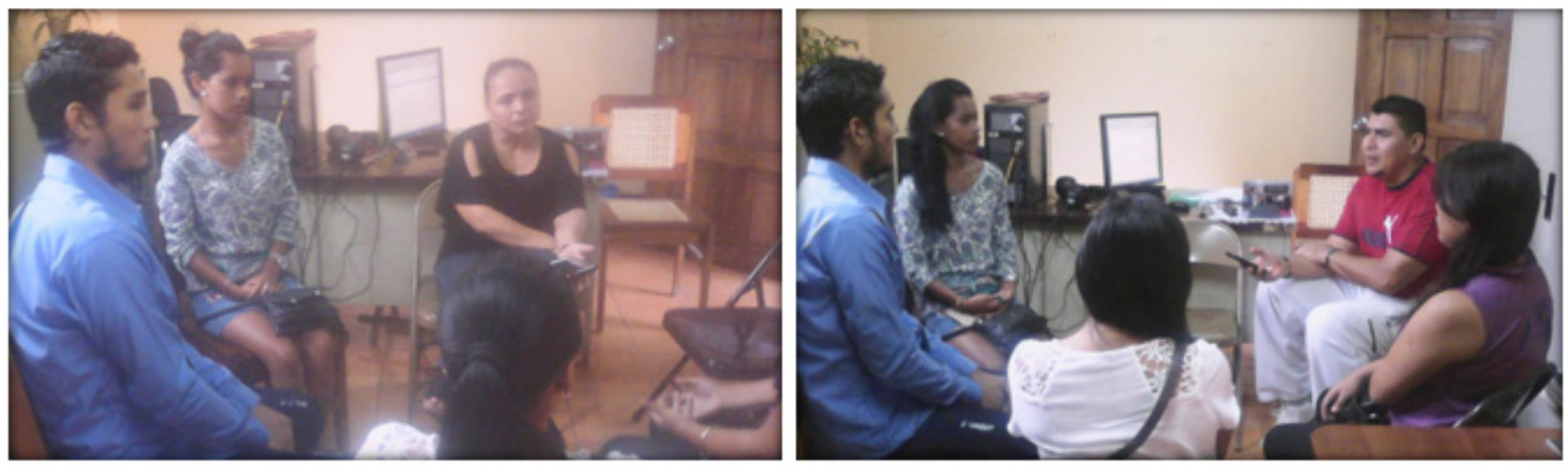

Fotografía del desarrollo del grupo focal con egresados
M. (2011). Modelo Educativo, Normativa y Metodología para la Planificación Curricular 2011. Managua: UNAN-Managua. 